\title{
Effect of Adjuvant Magnetic Fields in Radiotherapy on Non-Small-Cell Lung Cancer Cells In Vitro
}

\author{
Jianguo Feng, ${ }^{1,2}$ Huaying Sheng, ${ }^{1,2}$ Chihong Zhu, ${ }^{1,2}$ Hao Jiang, ${ }^{1}$ and Shenglin $\mathrm{Ma}^{3}$ \\ ${ }^{1}$ Cancer Research Institute, Zhejiang Cancer Hospital, No. 38 Guangji Road, Hangzhou, Zhejiang 310022, China \\ ${ }^{2}$ Key Laboratory Diagnosis and Treatment Technology on Thoracic Oncology, Hangzhou, Zhejiang 310022, China \\ ${ }^{3}$ Department of Oncology, Affiliated Hangzhou Hospital (Hangzhou First People's Hospital), Nanjing Medical University, \\ No. 261 Huansha Road, Hangzhou 310006, China
}

Correspondence should be addressed to Shenglin Ma; mashenglin65@gmail.com

Received 18 April 2013; Revised 12 July 2013; Accepted 12 July 2013

Academic Editor: Maria F. Chan

Copyright (C) 2013 Jianguo Feng et al. This is an open access article distributed under the Creative Commons Attribution License, which permits unrestricted use, distribution, and reproduction in any medium, provided the original work is properly cited.

Objectives. To explore sensitization and possible mechanisms of adjuvant magnetic fields (MFs) in radiotherapy (RT) of non-smallcell lung cancer. Methods. Human A549 lung adenocarcinoma cells were treated with MF, RT, and combined MF-RT. Colonyforming efficiency was calculated, cell cycle and apoptosis were measured, and changes in cell cycle- and apoptosis-related gene expression were measured by microarray. Results. A $0.5 \mathrm{~T}, 8 \mathrm{~Hz}$ stationary MF showed a duration-dependent inhibitory effect lasting for 1-4 hours. The MF-treated groups had significantly greater cell inhibition than did controls $(P<0.05)$. Surviving fractions and growth curves derived from colony-forming assay showed that the MF-only, RT-only, and MF-RT groups had inhibited cell growth; the MF-RT group showed a synergetic effect. Microarray of A549 cells exposed for 1 hour to MF showed that 19 cell cycleand apoptosis-related genes had 2-fold upregulation and 40 genes had 2-fold downregulation. MF significantly arrested cells in $\mathrm{G}_{2}$ and M phases, apparently sensitizing the cells to RT. Conclusions. MF may inhibit A549 cells and can increase their sensitivity to RT, possibly by affecting cell cycle- and apoptosis-related signaling pathways.

\section{Introduction}

Lung cancer is a common malignant tumor, and its incidence is rapidly growing: $64 \%$ of patients with non-small-cell lung cancer (NSCLC) need radiotherapy (RT); $45 \%$ of these patients receive primary RT. Although RT and chemotherapy together have better therapeutic effects, patients often cannot tolerate the toxicity and side effects of the combination. Optimizing treatment result is therefore critical.

Magnetic fields (MFs) are biologically effective, and their effect on tumors has been studied since the 1970s [1-5]. Although the mechanism of how MFs affect tumors is unclear, they have been shown to inhibit cancer cell growth and induce apoptosis. Magnetic fields influence charged particles. As such, they interfere with interactions among molecules and electrons in cells and possibly harm cellular functions such as DNA synthesis, thereby inhibiting cancer cell division and growth [6]. Zhang et al. reported that a $3 \mathrm{~Hz} /$ picosecond electromagnetic pulse can apparently inhibit growth of cervical carcinoma Hela cells by raising intercellular $\mathrm{Ca}^{2+}$ concentration, inducing apoptosis, and increasing Bax protein expression while decreasing $\mathrm{Bcl}-2$ expression (thus significantly increasing the $\mathrm{Bax} / \mathrm{Bcl}-2$ ratio) [7]. Lu et al. applied a low-frequency electromagnetic field on BEL-7402 hepatoma cells and found that expression of SODD and Survivin genes was significantly downregulated [8]. Wei et al. studied effects of rotational MFs combined with 5-fluorouracil (5-FU) on cell cycle and apoptosis in SP2/0 mouse myeloma cells, and found the S phase ratio was increased [9]. Magnetic fields alone cannot induce cell apoptosis, but they can sensitize cells to 5-FU toxicity, thus facilitating 5-FU-induced apoptosis. Liu et al. claimed that strong magnetic pulses significantly inhibited growth and exacerbated apoptosis in BIU-87 bladder carcinoma cells [10]. Pan et al. used microarray to measure and analyze the apoptosis-related gene-expression profile in MF-processed BEL-7402 hepatoma cells and L-02 fetus liver cells [11]. Electromagnetic field-processed cells upregulated expression 
TABLE 1: Inhibition rates under different magnetic field durations.

\begin{tabular}{lcc}
\hline Magnetic field duration $(\mathrm{h})$ & Absorbance (OD value) & Inhibition rate (\%) \\
\hline Control & $1.120 \pm 0.089$ & 0.0 \\
$1 \mathrm{~h}$ & $1.032 \pm 0.059$ & 7.9 \\
$\mathrm{~h}$ & $1.025 \pm 0.065$ & 8.5 \\
$3 \mathrm{~h}$ & $0.990 \pm 0.087$ & 11.6 \\
$4 \mathrm{~h}$ & $0.985 \pm 0.098$ & 12.1 \\
\hline
\end{tabular}

of apoptosis-inducing genes and downregulated expression of apoptosis-inhibiting genes. Han et al. used pulse MFs to study drug resistance in HL60/ADR leukemia cells [12]. Pulse MFs could downregulate MRP1 gene and protein expression, while increasing accumulation of cellular Rg123, and reverse multidrug resistance in leukemia cells.

Preliminary research showed that MFs, alone or together with chemotherapy, can inhibit tumor cell proliferation. However, few studies of MFs combined with RT in lung cancer are reported. We hypothesized that cell-cycle changes induced by MFs sensitize lung cancer cells to radiation. In this study, we designed experiments to measure the effect of adjuvant MFs in chemotherapy on colony formation, cell cycle, and apoptosis in A549 cells. Microarray was employed to elucidate the molecular and cellular mechanisms.

\section{Materials and Methods}

2.1. Cell Lines and Reagents. Lung adenocarcinoma cell line A549 was provided by Zhejiang Cancer Hospital. Cells were cultured in RPMI1640 media with 10\% bovine serum and kept in an incubator at $5 \% \mathrm{CO}_{2}$ and $37^{\circ} \mathrm{C}$ to promote growth. RPMI1640 was purchased from Gibco-BRL; bovine serum was purchased from HyClon.

2.2. Magnetic Field Duration and Radiation Dose. The inhibition rate was estimated by MTT assay to determine the duration of the MF effect. Using earlier research [13], 4 Gy was chosen as the radiation dose. Cells were transferred into 96-well plates at 500 cells/well and cultured for 24 hours. Four 8-well groups of cells were exposed to $0.5 \mathrm{~T}$ stationary MFs for 1, 2, 3, or 4 hours. After 48 hours, $20 \mu \mathrm{L} 5 \mathrm{mg} / \mathrm{mL}$ MTT was added into each well. After culturing for another 4 hours, supernatant was disposed, and $200 \mu \mathrm{L}$ was added into each well. After another 30 minutes, when brown crystals were completely dissolved, absorbance (AB) of each well was measured by enzyme-linked immunosorbent assay with $550 \mathrm{~nm}$ absorption wavelength. Inhibition rate of cell growth was calculated as [(Experimental AB - background control $\mathrm{AB}) /($ Control $\mathrm{AB}$ - background control $\mathrm{AB})] \times 100 \%$.

2.3. Colony-Forming and Surviving Curve Assay. Cells in logarithmic growth phase were digested into single-cell suspensions which were diluted and transferred into 6-well plates with 400 cells per well. After 24-hour adherent culturing, all cells were divided into 12 groups, each consisting of one plate of cells: one control group, five RT-only groups $(2,4,6,8$, or $10 \mathrm{~Gy}$ ), one MF-alone group ( $0.5 \mathrm{~T}, 8 \mathrm{~Hz}$ for 1 hour), and five
MF-RT combination groups $(0.5 \mathrm{~T}, 8 \mathrm{~Hz}$ for 1 hour; plus 2, 4, 6 , 8 , or $10 \mathrm{~Gy}$ ). Colonies were counted after 10 -hour culture. Colony-forming efficiency (CE) and surviving fraction (SF) were calculated with the following equations:

$$
\begin{gathered}
\mathrm{CE}=\frac{\text { Colonies observed }}{\text { Number of cells plated }} \\
\mathrm{SF}=\frac{\mathrm{CE} \text { of treated group }}{\mathrm{CE} \text { of control group }}
\end{gathered}
$$

Survival curves were drawn using multitarget single-hit models and linear quadratic models with SigmaPlot 10.0 software.

2.4. Superarray Gene Chip Assay. Cells at logarithmic growth phase were digested into single-cell suspensions, which were diluted and transferred into $75 \mathrm{~mL}$ culture flasks with $1 \times 10^{5}$ cells per flask. After 24-hour adherent culturing, three flasks of cells were exposed to $0.5 \mathrm{~T}, 8 \mathrm{~Hz} \mathrm{MF}$ for 1 hour, and three bottles of cells were used as controls. After another 24 hours of culturing, RNA was extracted for gene chip assays for each group.

2.5. Cell Cycle and Apoptosis Assay. Cells in logarithmic growth phase were digested into single cell suspensions, which were diluted and transferred into $25 \mathrm{~mL}$ culture flasks with $5 \times 10^{4}$ cells per flask. Cells were randomly divided into four groups: controls, MF-only group (0.5 T), RT-only group ( $4 \mathrm{~Gy})$, and combination group $(0.5 \mathrm{~T}+4 \mathrm{~Gy})$. Each group provided three parallel flasks for collection at 24,48 , and 72 hours separately. Cell cycle and apoptosis rates were measured by flow cytometry with an $\mathrm{ABC}$ cell cycle kit (BD Biosciences) and an Annexin V-FITC apoptosis detection kit.

2.6. Data Analysis. SPSS 11.0 software was used for statistical analysis. Measurement data are expressed as mean \pm standard deviation. Different groups were compared using one-way ANOVA. $P<0.05$ was considered statistically significant.

\section{Results}

3.1. Inhibition Rates under Different Magnetic Field Durations Measured with MTT Assay. The inhibitory effect of a $0.5 \mathrm{~T}$, $8 \mathrm{~Hz}$ stationary MF lasts for 1-4 hours, in a durationdependent manner (Table 1). Although the inhibitory effect did not significantly differ with magnetic duration $(P>0.05)$, MF-treated groups had significantly greater cell inhibition than the control group $(P<0.05)$. 
TABLE 2: Upregulated genes in A549 after 1-hour exposure to MF.

\begin{tabular}{lccc}
\hline Position & Genebank & Gene name & Fold change \\
\hline 138 & NM_003824 & FADD & XRCC1 \\
278 & NM_006297 & CDK8 & 4.32 \\
89 & NM_001260 & Rank & 3.42 \\
225 & NM_003839 & Cox-2 & 3.11 \\
199 & NM_000963 & CRAF1 & 2.87 \\
261 & NM_003300 & Fas/Apo-1/CD95 \\
236 & NM_000043 & DR3/Apo3 & 2.81 \\
227 & NM_003790 & Cyclin D1 \\
63 & NM_053056 & Cyclin C & 2.56 \\
62 & NM_005190 & TNFSF12/APO3L \\
244 & NM_003809 & Caspase 13 \\
43 & NM_003723 & Caspase 14 \\
44 & NM_012114 & Cyclin A1 \\
58 & NM_003914 & Mdm2 \\
182 & NM_002392 & Cyclin B2 \\
61 & NM_004701 & Cyclin G2 \\
70 & NM_004354 & Caspase-5 \\
48 & NM_004347 & Cyclin D2 & 2.48 \\
64 & NM_001759 & 2.37 \\
\hline
\end{tabular}

3.2. Colony-Forming Efficiency and Surviving Curve. The colony-forming assay showed that, for RT-only groups at 2, $4,6,8$, and $10 \mathrm{~Gy}$, the CEs were $16.4 \%, 13.2 \%, 10.2 \%, 7.1 \%$, and $1.2 \%$, respectively; SFs were $0.77,0.62,0.48,0.33$, and 0.24 , respectively. For MF-RT combined groups at 2, 4, 6, 8 , and $10 \mathrm{~Gy}$, CEs were $13.7 \%, 8.1 \%, 3.3 \%, 1.3 \%$, and $0.4 \%$, respectively, and SFs were $0.64,0.38,0.15,0.06$, and 0.02 , respectively. Cell survival decreased significantly $(P<0.05)$ with increasing RT dose in both RT groups and combination groups. Among groups with the same RT dose, the group with adjuvant MFs had a significantly smaller SF $(P<0.05)$, which suggests that A549 cells are more sensitive to RT with adjuvant MFs application. Survival curves are shown in Figure 1.

3.3. Gene Chip Assay. The microarray showed that after 1hour exposure to MFs, 19 cell cycle- and apoptosis-related genes in the A549 cells had 2-fold upregulation, and 40 genes had 2-fold downregulation (Tables 2 and 3). In particular, TNFRSF21 and CASPASE had significant upregulation, whereas expressions of ATM, p53, p57, p21, p27, TNFSF12, TNFRSF1OD, BAG4, BCL2L2, Mdn2, and XRCC1-5 were downregulated.

3.4. The Alternation of Cell Cycle and Apoptosis. Flow cytometry results showed that the MF-only group had $\mathrm{G}_{2}-\mathrm{M}$ phase arrest. Percentages of MF-only cells at $\mathrm{G}_{2}-\mathrm{M}$ were $24.2 \%$ for collection at 24 hours, $28.4 \%$ at 48 , hours and $18.5 \%$ at 72 hours-all significantly different from the control group. The MF-only group showed no significant difference in apoptosis index compared with the control group. Both the RT-only group and the MF-RT combination group showed significant apoptosis; however, the apoptosis index of combination

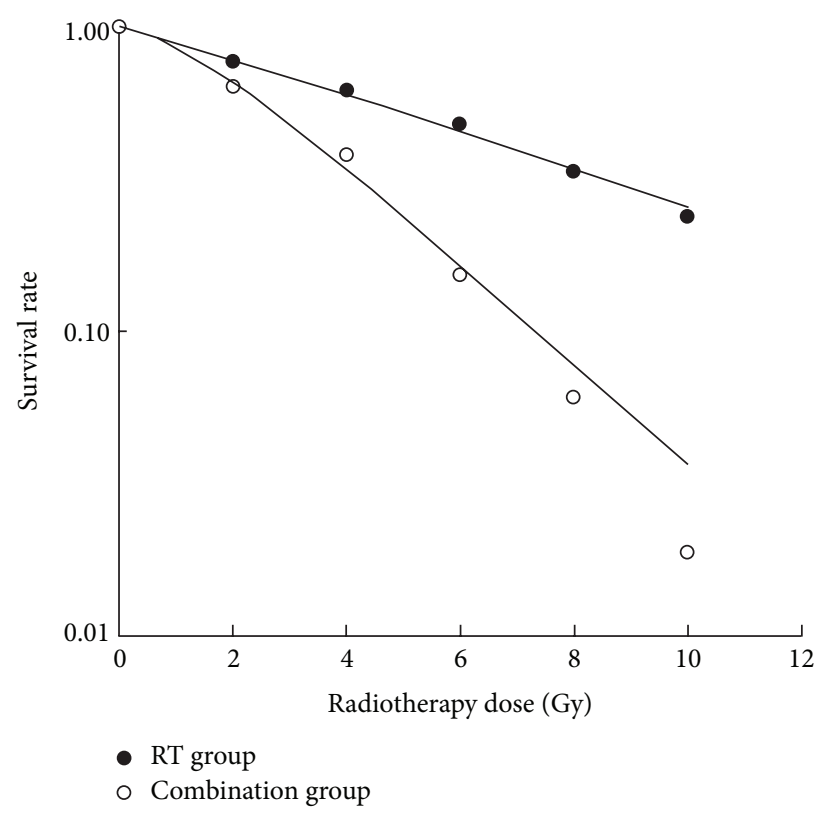

FIgURE 1: Cell survival rate after treatment with different dose of radiotherapy.

group was 34.6 for collection after 24 hours, which was significantly higher than that of the RT-only group (Figure 2 ).

\section{Discussion}

Repair of DNA double-strand breaks (DSBs) and cell-cycle regulation are two important factors that influence RT sensitivity of cells. ATM plays a very important role in DSB 
TABLE 3: Downregulated genes in A549 after 1-hour exposure to MF.

\begin{tabular}{|c|c|c|c|}
\hline Position & Genebank & Gene name & Fold change \\
\hline 228 & NM_003820 & TNFRSF14 & 0.03 \\
\hline 246 & NM_006573 & TNFSF13B & 0.09 \\
\hline 264 & NM_004620 & TRAF6 & 0.12 \\
\hline 96 & NM_001800 & p19-INK4D & 0.14 \\
\hline 232 & NM_001066 & TNFR2/p75 & 0.15 \\
\hline 86 & NM_000075 & Cdk4 & 0.17 \\
\hline 90 & NM_000389 & P21/Waf1/CIP1 & 0.18 \\
\hline 221 & NM_003844 & TRAIL-R/DR4 & 0.20 \\
\hline 88 & NM_001799 & CDK7 & 0.21 \\
\hline 128 & NM_001950 & $\mathrm{E} 2 \mathrm{~F}-4$ & 0.21 \\
\hline 229 & NM_001192 & TNFRSF17 & 0.22 \\
\hline 76 & NM_004358 & CDC25B & 0.23 \\
\hline 127 & NM_001949 & E2F-3 & 0.23 \\
\hline 282 & NM_021141 & KU80 & 0.26 \\
\hline 130 & NM_001952 & E2F-6 & 0.27 \\
\hline 135 & NM_005236 & XPF & 0.33 \\
\hline 204 & NM_000321 & $\mathrm{Rb}$ & 0.34 \\
\hline 114 & NM_000499 & CYP1A1 & 0.34 \\
\hline 262 & NM_004295 & TRAF-4 & 0.35 \\
\hline 122 & NM_004402 & DFF40/CPAN & 0.36 \\
\hline 26 & NM_004050 & Bcl-w & 0.38 \\
\hline 279 & NM_005431 & XRCC2 & 0.41 \\
\hline 7 & NM_000051 & ATM & 0.41 \\
\hline 17 & NM_001188 & Bak & 0.41 \\
\hline 132 & NM_001983 & ERCC1 & 0.42 \\
\hline 22 & NM_000633 & Bcl-2 & 0.42 \\
\hline 208 & NM_003804 & RIP & 0.43 \\
\hline 126 & NM_004091 & E2F-2 & 0.43 \\
\hline 142 & NM_001924 & GADD45 & 0.44 \\
\hline 91 & NM_004064 & p27Kip1 & 0.44 \\
\hline 71 & NM_001239 & Cyclin H & 0.45 \\
\hline 27 & NM_005178 & BCL-3 & 0.45 \\
\hline 281 & NM_003401 & XRCC4 & 0.46 \\
\hline 256 & NM_000546 & p53 & 0.46 \\
\hline 129 & NM_001951 & E2F-5 & 0.48 \\
\hline 242 & NM_003810 & TRAIL & 0.48 \\
\hline 125 & NM_005225 & E2F & 0.48 \\
\hline 240 & NM_001561 & $4-1 \mathrm{BB}$ & 0.49 \\
\hline 224 & NM_003840 & TRAIL-R4/DcR2 & 0.49 \\
\hline 220 & NM_000594 & TNFA & 0.50 \\
\hline
\end{tabular}

repair and cell cycle regulation signaling pathways. ATM activates the $G_{1}-S$ checkpoint by activating p53 and p 21 genes; it activates $S$ phase and $G_{2}-M$ checkpoints by activating the chk1, chk2, cdc25, and cdc2 genes [14]. When ATM expression is deficient or decreased, cell cycle checkpoints are dysfunctional, and cell cycle arrest is hindered. Thus, ATM expression and activity are related to RT sensitivity of cells [15]. In a study of sensitivity of nasopharyngeal carcinoma cell CNE-1 to RT, Hui et al. found that an RT sensitizing agent, UCN-01, works by weakening the cell's self-repair capability, and UCN-01 can only sensitize cells with p53 deficiency. Cyclin-dependent kinase inhibitor 1C (CDKN1C; p57, Kip2), which belongs to Cip/Kip family, can inhibit multiple $G_{1}$ cyclin/Cdk complexes and induce $\mathrm{G}_{1}$ arrest, thus inhibiting cell proliferation. CDKN1A (p21, Cip1) can inhibit CDK2 or CDK4 complexes and regulate the cell cycle. CDKN1A is regulated by $\mathrm{p} 53$ and can arrest cell in $\mathrm{G}_{1}$ phase under activating circumstances. CDKN1B (p27, Kip1), which encodes a CDKinhibitor protein, can inhibit activation of cyclin E/CDK2 or cyclin $\mathrm{D} / \mathrm{CDK} 4$ complexes and arrest the $\mathrm{G}_{1}$ phase as 

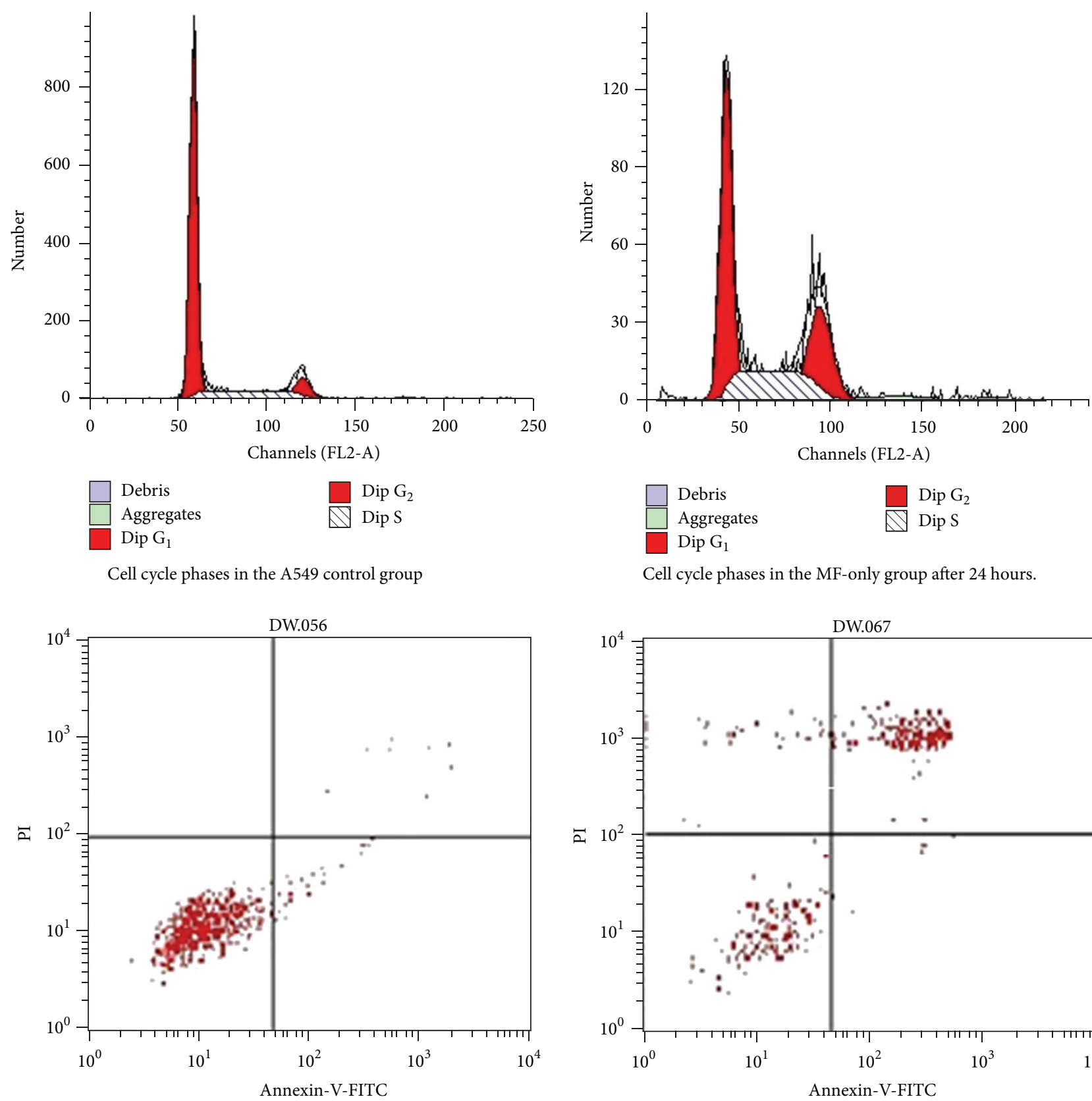

Apoptosis in the A549 control group

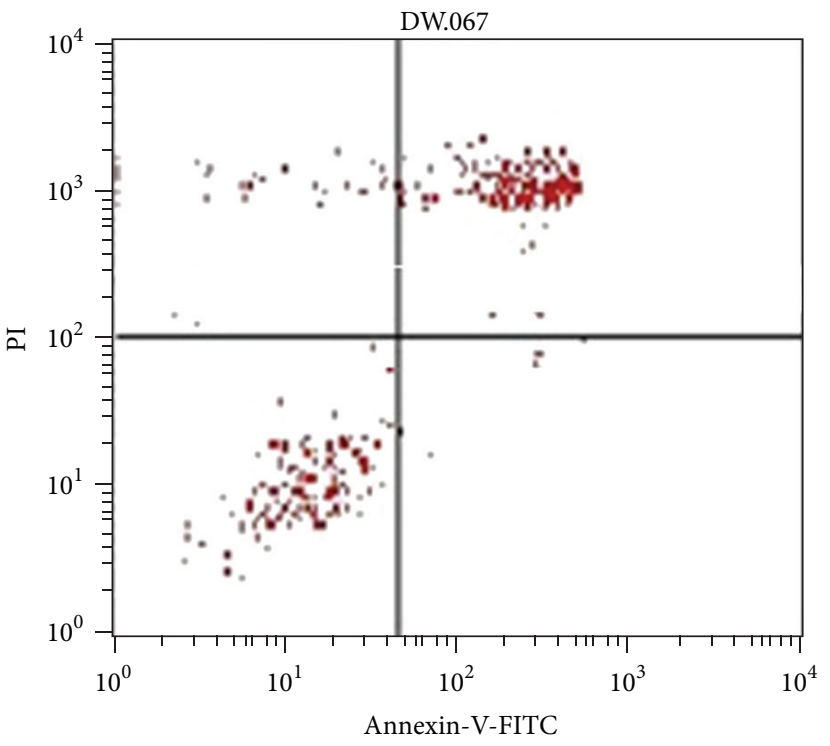

Apoptosis in the MF-only group after 24 hours

FIgURE 2: Changes in cell cycle and apoptosis.

well. TNFSF12, which belongs to TNF superfamily, can combine with the FN14/TWEAKR cytokine receptor, thus inducing apoptosis through multiple cell death pathways, and promote endothelial cell proliferation and migration (which are related to angiogenesis). TNFRSF21, whose functional domain activates the NF- $\kappa$ B and MAPK8/JNK pathways, also induces apoptosis. However, TNFRSF10D does not induce apoptosis and has been shown to play an inhibitory role in TRAIL-induced cell apoptosis. BAG4 is a member of the BAG1-related protein family. BAG4 is an antiapoptosis protein; it can interact with multiple apoptosis- and cell growth-related proteins, including BCL-2, Raf kinase, steroid receptor, growth factor receptor, and heat shock protein; it combines with TNFR1 and death receptor 3 to negatively regulate the downstream death signaling pathway. BCL2L2 belongs to the bcl-2 family; its expression induces apoptosis under cellular toxic environment. Mdn2 protein combines with and deactivates $\mathrm{p} 53$ and RB proteins, and it negatively regulates the $p 53$ gene. X-ray repair cross-complementing gene $(X R C C)$ is a major mediator of mammalian gene repair [16]. XRCC1, XPD, and XRCC3 proteins are the important components of BER, NER, and DSBR, respectively. XRCC1 repairs DNA single-strand breaks, induced by RT or alkylation agents, and works with DNA ligase III, polymerase 
beta, and poly(ADP-ribose) polymerase, involved in the BER pathway. XRCC2 and XRCC3 mediate RecA/Rad51-related proteins involved in homologous recombination to maintain chromosome stability and repair of double-strand breaks in DNA damage.

The gene chip results showed that, after MF exposure of A549 cells, the apoptosis-inducing gene TNFRSF21 was upregulated, as were several other apoptosis-related genes (e.g., ATM, p53, p57, p21, p27, TNFSF12, TNFRSF10D, BAG4, BCL2L2, Mdn2, and XRCC1-5). The upregulation of TNFRSF21 activated NF- $\kappa$ B and APK8/JNK pathways and induced apoptosis. Cellular sensitivity to RT is related to apoptosis rate [17]; higher apoptosis levels indicate higher sensitivity to RT, and rapidly apoptotic cells are more sensitive to RT. Conversely, downregulation of ATM and p53 increases apoptosis; downregulation of p57, p21, and p27 weakens cellcycle arresting function, thus inducing apoptosis; downregulation of antiapoptotic genes (TNFSF12, TNFRSF10D, BAG4, BCL2L2, and Mdn2) also induces apoptosis. Downregulation of XRCC1-5 also weakens DNA repair function, thus leading to cell death and weakened proliferative capacity.

Our study showed that, for a $0.5 \mathrm{~T}, 8 \mathrm{~Hz}$ stationary MF, duration had no significant effect $(P>0.05)$; however, groups treated with MF had significantly greater cell inhibition than controls $(P>0.05)$. The surviving fraction and growth curve derived from the colony-forming assay showed that MFonly, 4 Gy RT-only and the MF-RT combination groups had inhibited cell growth; the combination group in particular showed a synergetic effect $(P>0.01)$. The microarray showed that after A549 cells were exposed for 1 hour to MFs, 19 cell cycle- and apoptosis-related genes had 2-fold upregulation, especially TNFRSF21 and CASPASE, and 40 genes, including ATM, p53, p57, p21, p27, TNFSF12, TNFRSF10D, BAG4, BCL2L2, Mdn2, and XRCC1-5, had 2-fold downregulation. Magnetic fields significantly arrested cells in the $G_{2}$ and $M$ phases, which are the RT-sensitive phases; in this case, the cells were sensitized to RT. This study explored this sensitization effect and possible mechanisms of adjuvant MFs with RT on NSCLC at cellular and gene levels. Further study is needed to further clarify these mechanisms.

\section{Acknowledgments}

This work was supported by the Natural Science Foundation of Zhejiang Province (LY13H160028), Science and Technology Department of Zhejiang Province Key Scientific Research Projects for Social Development (2006C23018), and Zhejiang Provincial Medicine and Health Science Fund (2013KYA028).

\section{References}

[1] H. Chang, G. Li, G. Wang et al., "Report of 18 cases of the magnetic field therapy for malignant tumor," in Biomedical Physics Research, p. 74, Wuhan University Press, Wuhan, China, 1990.

[2] X. Xu, "Clinical observations of magnetotherapy on 10 patients with advanced malignancies," Chinese Biomagnetism, vol. 2, no. 1, p. 45, 1988.
[3] W. Zhao, "Four cases study on the lymphatic tumor treatment with the combination of gyromagnetic field and traditional Chinese medicine," Chinese Biomagnetism, vol. 5, no. 3-4, p. 124, 1991.

[4] H. Zhang, X. Zhang, C. Zhang et al., "Progress on the malignant tumor treatment with pulsed electromagnetic fields," Biomedical physics research. Atomic Energy Press, 19, 1992.

[5] F. Li, Q. Liu, Y. Ma et al., "Preliminary reports on the radioactive treatment of nasopharyngeal carcinoma with magnetic field," Chinese Biomagnetism, vol. 6, no. 1, p. 20, 1992.

[6] F. Zeng, "Experimental observation of the power-frequency magnetic field effects on mouse tumor," Biomagnetism, vol. 3, no. 4, p. 20, 1999.

[7] Y. Zhang, Z. Xiong, Y. Hua et al., "In vitro study of damaging effects of intense picosecond pulsed electric field on HeLa cells," Chongqing Medicinal Journal, vol. 41, no. 18, pp. 1785-1791, 2012.

[8] X. Lu, W. Qian, C. Xia et al., "Effects of ELF on gene expression of SODD and Survivin in hepatoma cell lines," Journal of Soochow University, vol. 31, no. 5, pp. 723-726, 2011.

[9] S. Wei, C. Zhang, X. Sha et al., "Effect of rotary magnetic field combining 5-fluorouracil on cell cycle and apoptosis in SP2/0 cell line," Cancer Research on Prevention and Treatment, vol. 37, no. 12, pp. 1367-1369, 2010.

[10] Q. Liu, Y. Ma, J. Li et al., "Effects of high pulsed magnetic field on apoptosis of human bladder cancer BIU-87 cells," Journal of Clinical Urology, vol. 25, no. 7, pp. 550-552, 2010.

[11] J. Pan, W. Zhao, J. Wen et al., "Effects of electromagnetic field on gene expression associated with cellular proliferation and apoptosis in hepatoma cell lines," Jiangsu Medical Journal, no. 6, pp. 637-639, 2009.

[12] Q. Han, X. Liu, Y. Han et al., "Mechanism of pulsed magnetic field on reversal of multi-drug resistance of the leukemic cell HL60/ADR," Progress in Modern Biomedicine, vol. 9, no. 1, pp. $1-4,2009$.

[13] H. Jiang, S. Ma, and J. Feng, "In vitro study of radiosensitization by $\beta$-Elemene in A549 cell line from adenocarcinoma of lung," Chinese Journal of Radiological Medicine and Protection, vol. 29, no. 04, pp. 395-396, 2009.

[14] P. J. McKinnon, "ATM and ataxia telangiectasia. Second in molecular medicine review series," EMBO Reports, vol. 5, no. 8, pp. 772-776, 2004.

[15] M. B. Kastan, D.-S. Lim, S.-T. Kim, and D. Yang, "ATM-a key determinant of multiple cellular responses to irradiation," Acta Oncologica, vol. 40, no. 6, pp. 686-688, 2001.

[16] J. Thacker and M. Z. Zdzienicka, "The mammalian XRCC genes: their roles in DNA repair and genetic stability," DNA Repair, vol. 2, no. 6, pp. 655-672, 2003.

[17] X. Xu and S. Yu, Modern Radiation Oncology, people's Military Medical Press, Peking, China, 2000. 


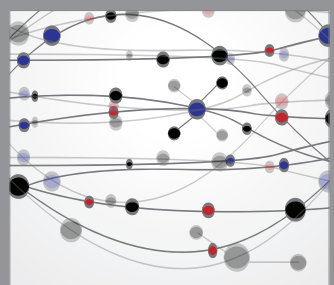

The Scientific World Journal
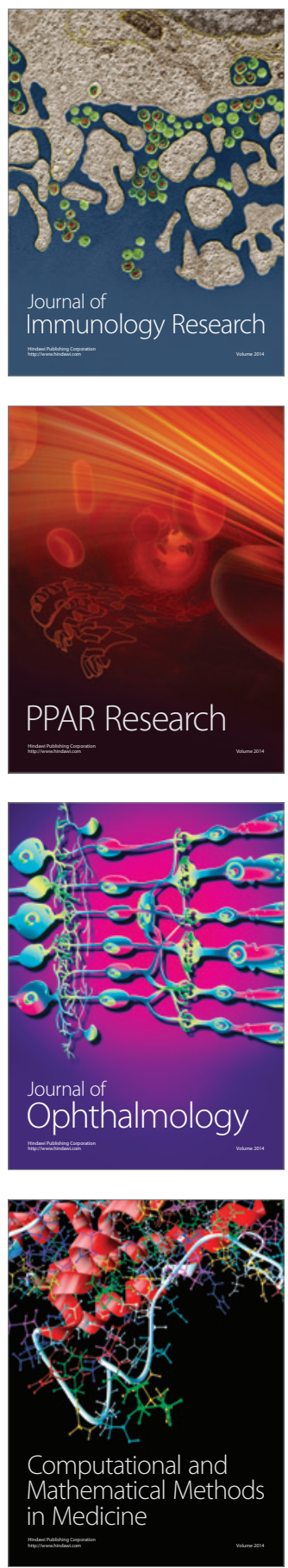

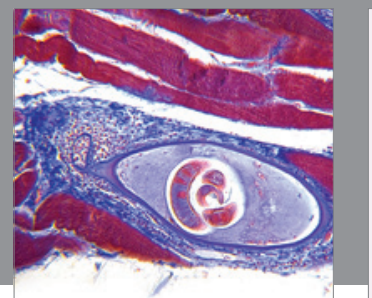

Gastroenterology

Research and Practice
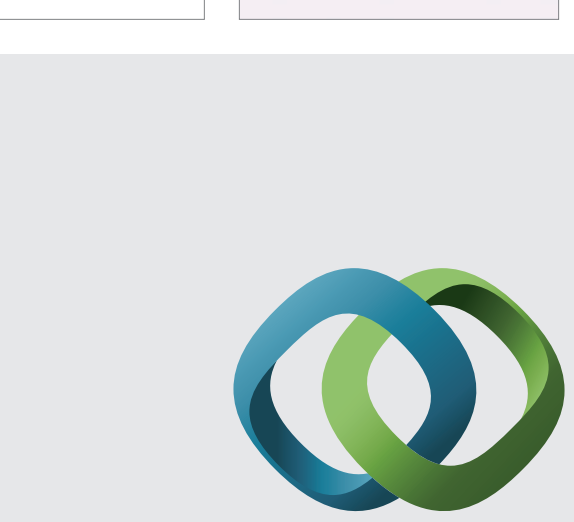

\section{Hindawi}

Submit your manuscripts at

http://www.hindawi.com
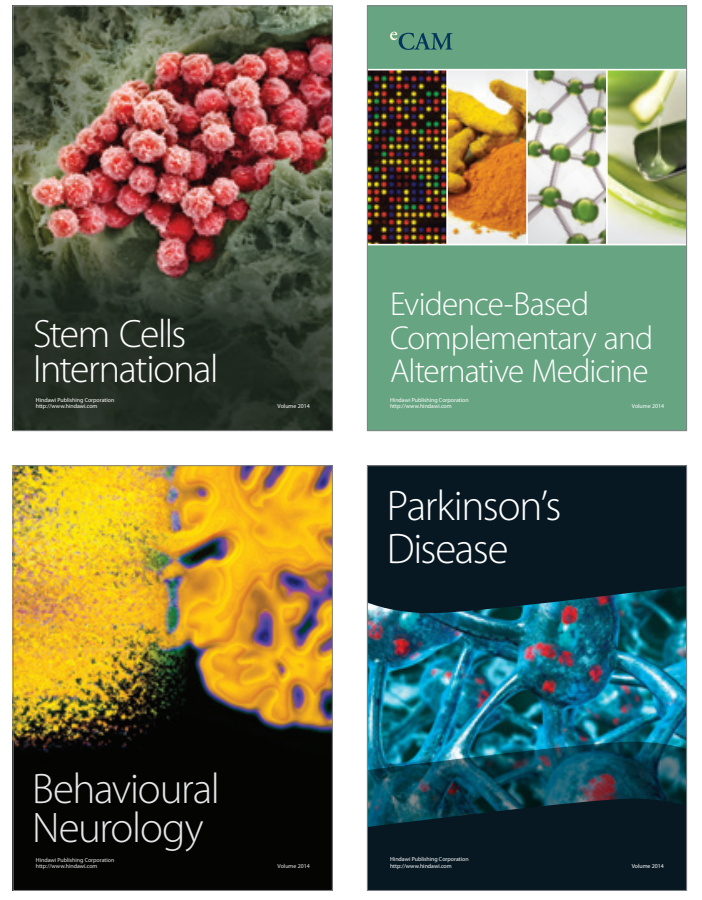
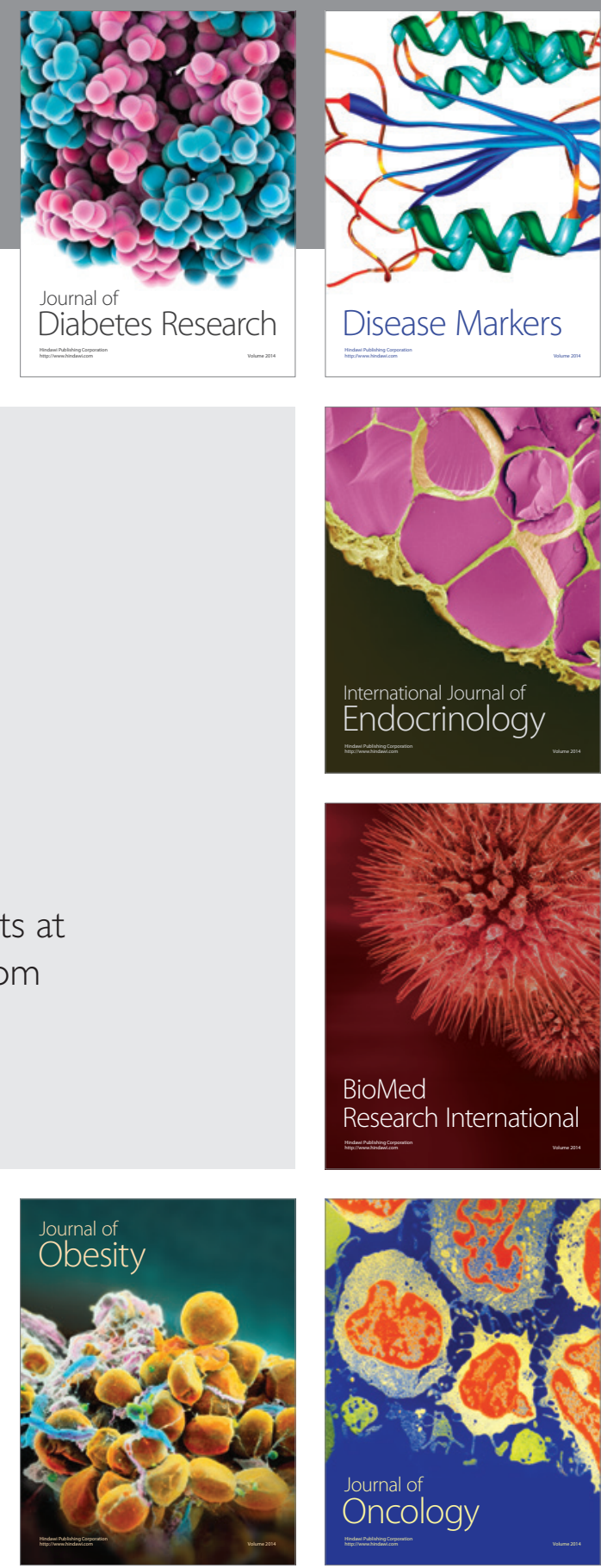

Disease Markers
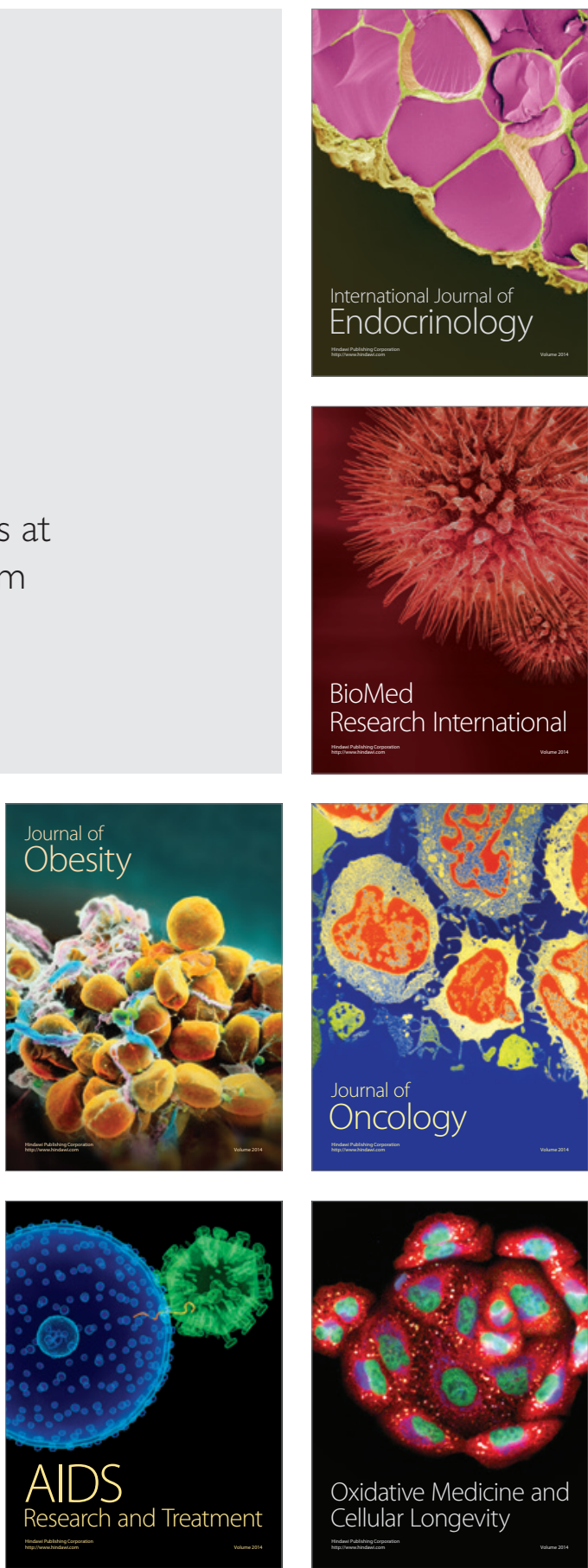